\title{
Inter-relationship of soft contact lens diameter, base curve radius, and fit
}

\begin{abstract}
PURPOSE: To evaluate the inter-relationship of soft contact lens base curve radius (BC), diameter and lens fit using a mathematical model.

METHOD: A spreadsheet mathematical model was used to evaluate theoretical fitting characteristics for various combinations of soft lens BC and diameter. The designs were evaluated using ocular topography data collected from 163 U.K. subjects. The model evaluated lens tightness (edge strain) and on-eye diameter (horizontal corneal overlap) and assumed that acceptable values fell within the range 0 to $6 \%$ and 0.2 to $1.2 \mathrm{~mm}$, respectively. Analyses were undertaken of various trends relating to soft lens fit, including: i) the effect of $B C$ and diameter on fitting success, ii) the effect of lens asphericity, $\mathrm{BC}$ and sag on lens diameter on the eye, iii) the effect of lens diameter on lens tightness.
\end{abstract}

RESULTS: The highest overall success rate (90.2\%) was achieved with an 8.60/14.2 mm (BC/diameter) design. Using this design on the sample population, the median edge strain value was 3.2\% (IQR: $2.1 \%$ ) while median corneal overlap was $0.62 \mathrm{~mm}$ (IQR: 0.35 ). There was a positive correlation $(r=0.37, p<0.0001)$ between edge strain and corneal overlap. Edge strain showed significant correlations with each of the ocular topography variables, most notably corneal asphericity $0.62, p<0.0001)$. Corneal overlap showed significant correlations with corneal asphericity $(r=-0.42$, $p<0.0001)$ and corneal diameter $(r=-0.92, p<0.0001)$. For a $0.4 \mathrm{~mm}$ change in $B C$, it is necessary to change diameter by $0.2 \mathrm{~mm}$ to maintain similar on-eye diameter (arclength). When changing lens diameter, a change in $\mathrm{BC}$ of $0.2 \mathrm{~mm}$ is required in order to maintain similar tightness of fit.

CONCLUSIONS: Mathematical modelling is a useful technique for large scale evaluation of the interactions of soft contact lens design and fit. The study has given useful insights into the general performance of soft lens designs.

Keywords: soft contact lens, diameter, base curve radius, tightness, corneal coverage

\section{INTRODUCTION}

Since their first development, attempts have been made to correlate soft contact lens fitting characteristics with physical parameters but with only limited success. One problem is that it is logistically difficult to assess a wide range of soft lens designs on a wide range of eyes. An obvious alternative to clinical testing of soft lens fit is to use computer modelling. This is routinely used in the commercial development of soft lens designs and recently a spreadsheet model has been proposed. ${ }^{1}$ This is less sophisticated than some proprietary software but has the advantages of being versatile, fast, and in the public domain.

Soft contact lens fit is important in maximising comfort and minimising any effects on ocular physiology. ${ }^{2}$ Various aspects of soft lens fit are conventionally assessed; however, arguably the two most important 
characteristics are lens tightness and corneal coverage. Lens tightness is typically assessed by the push-up test and has been shown to be the most reliable predictor of overall fit ${ }^{3}$ and is assumed to be governed by lens base curve radius $(\mathrm{BC})$. Complete, corneal coverage is necessary for a comfortable fit that avoids mechanical irritation of the cornea from the lens edge. Whether there is adequate corneal coverage can be assessed by measuring the amount that the lens overlaps the limbus and this is assumed to be governed by the lens' diameter. Lens centration, movement and edge alignment are also cited as important lens characteristics but these are subsidiary to lens tightness.

Modifying either BC or diameter will have an effect on both of these key aspects of lens fit. For instance, making a large increase in $\mathrm{BC}$ but no change in diameter will loosen the fit but will also result in a smaller on-eye diameter since the lens surface area is reduced.

Several analyses have attempted to evaluate the combined effect of BC and diameter by considering lens sagittal depth and comparing this with the corresponding ocular sagittal depth. ${ }^{4-8}$ There are several limitations to this approach. First, ocular sagittal depth does not accurately characterise variations in corneal profile. Two eyes of similar sagittal depth (for a given chord diameter) might give different fitting characteristics for a given lens due to differences in corneal asphericity, corneal diameter and corneoscleral junction profile. Similarly, two lenses of similar sagittal depth may show differences in fit due to differences in surface area arising from differences in profile, for instance due to differences in lens asphericity. Furthermore, the sagittal depth of the lens changes considerably when fitted to the eye. Some stretching of the lens periphery and, therefore, reduction in sagittal depth is usually necessary to achieve a satisfactory fit. ${ }^{4}$

A potentially better approach to modelling soft contact lens fit is to consider lens centre to edge arclength and to compare it with that of the eye. A previous study has employed this technique to calculate stretching of the lens edge which is assumed to correlate with lens tightness of fit. ${ }^{1}$ This gave insights into the effect of lens design and ocular parameters on lens tightness. By systematically varying a single parameter it was possible to assess the effect of specific changes on lens fit.

The previous study only evaluated lens fit in relation to tightness, however, the model can also estimate, for a given eye shape, the amount by which a specific lens design overlaps the cornea, thus, modelling the important characteristic of corneal coverage. This allows a better evaluation of the effect of changes in lens diameter and BC and their interrelationship.

The previous study focused on a single model eye and single representative lens design. A further refinement of the modelling technique is to assess lens designs on a range of ocular topographies to assess their effect on a representative population. This latter approach, therefore, allows for the true range and distribution of ocular topographies within the population. It allows an estimation of the fitting success rate for a given lens design on a typical population.

The purpose of this study has been to use computer modelling to evaluate various properties of soft contact lens design, in particular, the interaction of $\mathrm{BC}$ and diameter and their joint influence on lens fit.

\section{METHOD}

This computer modelling study estimated the effects of varying soft contact lens BC and diameter on the two key aspects of lens fit: tightness and corneal overlap. Theoretical fitting success rates were calculated using population data from a previous study. ${ }^{10} \mathrm{~A}$ spreadsheet computer model was used to calculate soft lens fit characteristics against pre-set acceptance criteria and to determine whether given lenses on given eyes were acceptable. The spreadsheet is a refinement of a computer model 
previously reported in this journal. ${ }^{1}$ Whereas the original model was rotationally symmetric, this updated version allows separate inputs for vertical and horizontal ocular topography.

The lens inputs were $\mathrm{BC}$ and diameter. The ocular topography inputs were corneal apical radius, corneal asphericity (shape factor, 1- $\mathrm{e}^{2}$ ), corneal diameter and corneoscleral junction angle in the horizontal and vertical meridians. Corneal diameter was the true diameter, measured from sulcus to sulcus, as opposed to the conventional clinical measurement of horizontal visible iris diameter (HVID) which underestimates corneal diameter by approximately $1.5 \mathrm{~mm} .{ }^{10,11}$

Briefly, the model works by calculating the on-eye diameter of the lens having assumed that the back surface of the lens is perfectly aligned to the ocular surface and that there is no change in the arclength of the lens. ${ }^{12}$ The model therefore assumes that alignment is achieved by flexing of the lens radially and stretching of the lens circumferentially. The model takes no account of lens thickness, front surface design or material properties.

Since the percentage change in lens circumference is the same as the percentage change in lens diameter, the stretching of the lens edge (edge strain) is calculated by comparing the off-eye diameter with the on-eye diameter (Fig. 1). Corneal overlap is calculated by subtracting the horizontal corneal diameter from the lens' horizontal on-eye diameter.

For a given lens design and eye, the model determined whether a given lens provided an acceptable fit based on two characteristics: i) edge strain (tightness) and ii) horizontal corneal overlap. The edge strain was averaged from the calculations for horizontal and vertical meridians. The lens' overlap of the cornea was based on only the horizontal meridian since the horizontal cornea is invariably larger than the vertical. ${ }^{10}$ For a lens to be judged acceptable, it was required to show mean edge strain falling within the range 0 to $6 \%$ and horizontal corneal overlap falling within the range 0.2 to $1.2 \mathrm{~mm}$.

As with edge strain, the change in lens sagittal depth was calculated as a percentage of the original and averaged for the horizontal and vertical meridians.

\section{Subject Database}

To assess the fitting characteristics of various lens designs, a representative population database was used which comprised ocular topography data from 163 eligible subjects who had been examined as part of a previous study undertaken in the U.K. (Table 1). ${ }^{10}$ This previous study sought to provide normative data on corneoscleral topography in a healthy, visually-normal population. Subjects were measured using a Medmont E300 corneal topographer (Medmont, Camberwell, Australia) for corneal apical radius and shape factor in the horizontal and vertical meridians. A Visante anterior segment ocular coherence tomographer (Carl Zeiss Meditec, Dublin, CA) was used to measure true corneal diameter and corneoscleral junction angle in the horizontal and vertical meridians.

To be included in the database, subjects were required to have spectacle astigmatism less than or equal to $2.00 \mathrm{D}$ and no previous corneal refractive surgery or other severe corneal irregularity. Ocular topography data from only one eye per subject were used; these were the right eye data unless only the left eye data were available.

\section{Lens Fit Analysis}

Analyses were undertaken to evaluate various trends relating to soft contact lens fit. In the first part, the variability of lens fit within a representative population was investigated. In the second part, ranges 
of lens design showing equivalent on-eye lens fit were investigated. Specifically, the following evaluations were undertaken:

i) The effect of $\mathrm{BC}$ and diameter on fitting success

ii) The distribution of lens fittings within the population

iii) Correlations between lens fit and ocular topography

iv) Correlation between change in lens diameter and sagittal depth

v) The effect of $B C$ on on-eye diameter

vi) The effect of diameter changes on lens tightness

vii) The effect of lens asphericity on on-eye diameter

For a given lens design, the success rates were based on the proportion of test eyes showing an acceptable fit for tightness, diameter acceptance and overall acceptance. The overall success rate, therefore, indicated the proportion of lens fittings that were acceptable for both diameter and tightness. The lens design was a simple spherical monocurve back surface as this is the design most commonly used with current soft lenses. For the most successful BC-diameter combination, frequency distribution curves were plotted for edge strain and corneal overlap.

The effect of variation in $\mathrm{BC}$ was evaluated by calculating lenses of equivalent arclength and, therefore, equivalent on-eye diameter. Lens centre to edge arclength was calculated using the following formula:

$$
A L=A S I N((D / 2) / B C) \times B C
$$

Where: $\mathrm{D}=$ lens diameter $\mathrm{BC}=$ base curve radius

The effect of variation in diameter on lens fit was evaluated using the population database to find BCdiameter variations giving the same mean edge strain. These were plotted for three values of edge strain: $0 \%, 3 \%$, and $6 \%$. The middle value $(3 \%)$ is assumed to correspond to optimum lens tightness while $0 \%$ and $6 \%$ are assumed to be the thresholds for loose and tight lenses, respectively. These edge strain thresholds are assumptions based on the range of fittings seen in a typical population with a typical soft lens design and the expected proportion of tight and loose fittings. ${ }^{1}$ The lower threshold for corneal overlap $(0.2 \mathrm{~mm})$ is a conservative estimate based on the requirement for complete corneal coverage. The upper threshold is an assumption, again, based on the range of fittings seen in a typical population.

Though the basic model utilizes a spherical lens back surface, it was possible to study the effect of back surface asphericity by calculating the lens centre to edge arclength while varying sagittal depth. This was achieved by varying the lens' shape factor $(0.7$ to 1.3$)$ for a given lens diameter. This was undertaken using a refinement of the spreadsheet computer model which uses an iterative calculation in which the ellipse is divided into $0.05 \mathrm{~mm}$ sections and Pythagoras' theorem is used to approximate each arclength. 


\section{Data Analysis}

Having tested for normal distribution, associations between selected variables were tested using Pearson's correlation coefficient. A Benjamini and Hochberg correction was used to control the false discovery rate for multiple comparisons and preserve the family-wise $5 \%$ type I error rate. ${ }^{13}$

\section{RESULTS}

\section{Variability of Lens Fit}

The effect of varying lens diameter and $\mathrm{BC}$ on overall success rate was evaluated for a spherical monocurve design (Table 3,). The highest overall success rate (90.2\%) was achieved with an 8.60/14.2 $\mathrm{mm}$ (BC/diameter) design. Relatively high success rates (>85\%) were achieved across a range of BCdiameter combinations: for instance, $8.60 / 14.0 \mathrm{~mm}$ to $8.80 / 14.6 \mathrm{~mm}$. Outside of this, there was a sharp fall in success rates.

Success rates were also calculated based on tightness (edge strain) alone, i.e. ignoring diameter acceptance. The highest success rate based on tightness alone $(97.5 \%)$ was achieved with an $8.40 / 13.6 \mathrm{~mm}$ design, however, this success rate was close to that achieved with the most successful design for overall fit $(96.9 \%)$.

Using the most successful design $(8.60 / 14.2 \mathrm{~mm})$, the distribution of lens fitting characteristics was evaluated on the sample population (Fig. 2, Fig. 3). The median edge strain value was $3.2 \%$ (IQR $2.1 \%$ ) while the median corneal overlap was $0.62 \mathrm{~mm}$ (IQR 0.35). The scatterplot in Fig. 4 shows a positive correlation $(r=0.37, p<0.0001)$ between edge strain and corneal overlap, with greater overlap being associate with greater edge strain. Those fittings which were relatively large on-eye were also relatively tight.

Correlations between lens fit and ocular topography variables are summarised in Table 2. Edge strain showed significant correlations with corneal asphericity $(+0.52, p<0.0001)$ and apical radius $(r=-0.60$, $p<0.0001$ ). Increasing tightness (edge strain) was, therefore, associated with flatter apical radius and increasing prolate corneal asphericity (Fig. 5). Corneal overlap showed a significant correlation with corneal asphericity $(r=-0.42, p<0.0001)$ and corneal diameter $(r=-0.92, p<0.0001)$. Greater corneal overlap (i.e. larger lenses on-eye) was associated with increasing prolate corneal asphericity and, as expected, with decreasing corneal diameter.

The median reduction in lens sagittal depth was 4.5\% (IQR: 5.8\%). The scatterplot in Fig. 6 shows a positive correlation $(r=+0.97, p<0.0001)$ between edge strain and reduction in lens sagittal depth. The curve intercepts zero change in sagittal depth when edge strain is approximately $1.5 \%$. For the range of acceptable edge strain $(0-6 \%)$, the decrease in lens sagittal depth ranged between $-3 \%$ and $+13 \%$.

\section{Equivalent On-eye Fittings}

For a given lens diameter, changing the base curve changes the lens' surface area and, thus, a steeper lens of given diameter will appear larger on the eye than a flatter lens of similar diameter. In order to preserve a similar on-eye diameter, when flattening BC, it is therefore, necessary to increase the diameter to compensate. Fig. 7 Illustrates lenses of equivalent radial arclength; the curves, therefore, 
show the changes in diameter required for varying $\mathrm{BCs}$ to maintain a constant on-eye diameter. A change in $\mathrm{BC}$ of $0.4 \mathrm{~mm}$ results in a change in on-eye diameter of approximately $0.2 \mathrm{~mm}$.

Increasing the diameter of a lens while maintaining the same BC results in a tightening of fit (edge strain). Fig. 8 shows lenses of equivalent mean tightness. The data were obtained by using the model and subject database to determine mean edge strain for varying lens designs. The curves show that, when increasing lens diameter by $0.4 \mathrm{~mm}$, it is necessary to flatten the $\mathrm{BC}$ by $0.2 \mathrm{~mm}$ in order to maintain the same tightness of fit.

Another variable that can affect on-eye diameter is lens asphericity. For a given sagittal depth, a lens incorporating a steepening periphery design (oblate) will have a larger surface area than a spherical design and, therefore, can be expected to show a larger on-eye diameter. Fig. 9 shows the variation in lens arclength with lens asphericity for various lens sagittal depths. In the range shown (shape factor: 0.7 to 1.3), the variation in lens asphericity results in a change in lens arclength (or on-eye diameter) of approximately $0.1 \mathrm{~mm}$.

\section{DISCUSSION}

In contrast to previous work ${ }^{1}$, this study has used a mathematical model to evaluate variation in lens fit in a representative range of ocular topographies, rather than using a single model eye. The study has also expanded the evaluation of lens fit by considering diameter acceptance, in the form of corneal overlap, as well as lens tightness. The technique allows testing of an extensive range of lens designs on a scale that would be impractical in a clinical study. The study has given a number of useful insights into the general performance of soft lens designs that, otherwise, would have required a multiple clinical studies. A better understanding of the interaction between soft lens diameter, BC, and fit is useful to both practitioners and lens designers.

Evaluating the effect of lens design suggests that a single BC-diameter combination can achieve a success rate of approximately $90 \%$. A high level of success (>85\%) was achieved with a relatively small range of designs (Table 3) most of which are not represented by marketed lens designs. The model assumed no shrinkage but, with actual lenses, the optimum design $(8.60 / 14.2 \mathrm{~mm})$ would need to be larger and flatter to compensate for on-eye shrinkage. ${ }^{14,15}$ With the most temperature sensitive materials, such as high water non-ionic Group 2 materials, this would equate to approximately 9.00/15.0 $\mathrm{mm}$ (BC/diameter).

When fitted to a representative population, even the most successful design produces a wide range in lens fitting characteristics as evidenced by the wide distributions of edge strain (Fig. 2) and corneal overlap (Fig. 3). This is to be expected given the wide range in ocular topographies. It emphasises the need for manufacturers either to provide multiple fittings or at least to select a single, versatile design that sits in the high success zone shown in Fig. 1.

These analyses suggest that lenses which are relatively large on-eye are also likely to be relatively tight fitting. In Fig. 4, all but two of the lenses with $>1.0 \mathrm{~mm}$ overlap are tighter than the theoretical optimum value of $3 \%$. Conversely, all of the fittings with $<0.3 \mathrm{~mm}$ overlap are looser than the theoretical optimum value. The clinical consequences of small lenses that fail to give full corneal coverage are well understood. Pressure from the lens edge is likely to irritate the cornea causing discomfort and corneal staining. The consequences of excessively large lenses are less well understood, however, even 
borderline tight lens fittings have been shown to produce more corneal and conjunctival staining than optimally fit lenses. ${ }^{16}$

Given the nature of the calculations, it is not surprising that there were strong correlations between the input and output variables. Nevertheless, the correlations provide interesting insights into the relative importance of the various ocular topography variables. Once again, the findings emphasise that attempting to predict lens fit from keratometry alone is simplistic and that this is one of at least three ocular parameters that are influential. The importance of corneoscleral junction profile in relation to lens fit has been much discussed ${ }^{17,18}$ but the findings of this study suggest that, for most patients, this is of minimal significance. A previous evaluation suggested that the greatest influence from corneoscleral profile was with eyes showing a small corneal diameter and sharp corneoscleral junction. ${ }^{1}$

One of the strongest correlations between lens fit and ocular topography indicated increasing tightness (edge strain) with increasing corneal prolate asphericity. This is consistent with a previous clinical study in which increasing corneal prolate asphericity was associated with greater lens movement and decentration. ${ }^{19}$ The relationship between these two fit variables and tightness of fit is not linear, ${ }^{20}$ however, looser lens fittings tend to show more movement and decentration. ${ }^{3}$

Previous workers have assumed that the goal of soft lens fitting is to match the sagittal depth of the lens with the corresponding sagittal height of the eye. ${ }^{5-8}$ The present analysis suggests that, for most fittings, there is a shortening of sagittal depth when the lens is applies to the eye. With a typical optimally fitting lens (i.e. edge strain $3 \%$ ), the sagittal depth decreases by approximately $5 \%$. Interestingly, with some looser fittings, the on-eye sagittal depth of the lens is unchanged or even increased; this is due to the steepening of the lens to match the central cornea being counteracted by flattening of the lens over the peripheral cornea and the sclera.

Calculations of equivalent on-eye fittings give useful indications as to how optimal fittings can be achieved. For instance, for lenses of optimal size but showing excessive tightness, it is necessary to increase diameter as well as flatten $B C$ to loosen the fit and maintain the same on-eye diameter. In Fig. 7, this equates to moving upwards in accordance with one of the curves. For a lens showing optimum tightness but inadequate size, it is also necessary to increase the diameter and BC but in a different proportion. In Fig. 8 this equates to moving rightwards in accordance with one of the curves, to give a larger lens but with similar tightness of fit.

One concern with this method of computer modelling lens fit is that it assumes spherical back surface lens designs and, therefore, does not take account of any asphericity. The analysis in Fig. 9 shows that the effect is relatively minor; the difference in on-eye diameter between a spherical and typical prolate soft lens design is about $0.07 \mathrm{~mm}$.

\section{Limitations}

Inevitably with mathematical modelling, there are various limitations to the methodology. The thresholds for tight and loose lens fittings are assumptions based on a comparison of data from the model and expectations from clinical experience. The model makes no allowance for variation in modulus or lens thickness. It is likely that the thresholds will vary according to modulus, ${ }^{21,22}$ with higher modulus materials resulting in a narrower range of acceptable fits. The model results apply only to a single, non-specific set of material properties and, therefore, outcomes for actual lenses may differ from these.

The ocular topography data were from a predominantly Caucasian population. Since some variations in ocular topography have been noted between racial and even ethnic groups, ${ }^{23}$ it is possible that the 
success rates and distribution of lens fittings would vary between ethnicities. This technique of large scale modelling is particularly suitable for comparing lens performance between ethnic groups but requires comprehensive datasets of ocular topography.

The calculations of diameter acceptance assume no decentration. With lens fittings that have minimal corneal overlap, decentration could make the difference between an acceptable and unacceptable fit. Lens centration is considered a key fitting characteristic and is systematically evaluated during contact lens fitting assessments, however, decentration tends to be a secondary characteristic deriving from a loose fit. Similarly, edge fit is an important fitting characteristic but edge stand-off tends to derive from loose fittings in which the diameter of the lens is wider than that of the eye and, therefore, no stretching of the edge is required. On the other hand, excessive edge tightness, characterised by conjunctival indentation, derives from excessive overall tightness.

The model assumes that lenses align with the front surface of the eye. Previous ocular coherence tomography (OCT) studies have noted that a gap can form between the limbus and the lens. ${ }^{24,25}$ This would be particularly relevant in cases of a sharp corneoscleral junction fitted with a lens of relatively high stiffness. However, theoretical calculations (not detailed here) suggest that this would have only a small effect on corneal overlap calculations $(\approx 0.05 \mathrm{~mm})$.

\section{CONCLUSIONS}

Mathematical modelling is a useful technique for evaluating soft lens fitting characteristics for a wide range of lens designs on a large population. When evaluating the effect of varying $B C$ and diameter in tandem, the calculation of arclength is a more useful approach than sagittal depth. When using a single lens design on a wide ranging population, lenses which show greater corneal overlap tend to be tighter. This evaluation has also highlighted some 'rules of thumb' for the interaction of $\mathrm{BC}$, diameter and soft lens fit. For a $0.4 \mathrm{~mm}$ change in $\mathrm{BC}$, it is necessary to change the diameter by $0.2 \mathrm{~mm}$ to maintain similar on-eye diameter. When changing diameter by $0.4 \mathrm{~mm}$, a change in $\mathrm{BC}$ of $0.2 \mathrm{~mm}$ is required in order to maintain similar lens tightness. 


\section{REFERENCES}

1. Young G. Mathematical model for evaluating soft contact lens fit. Optom Vis Sci 2014;91:16776.

2. Jones L, Brennan N, Gonzalez-Meijome J, Lally J, Maldonado-Codina C, Schmidt T, Subbaraman L, Young G, Nichols J. The international workshop on contact lens discomfort: Report of the contact lens materials, design \& care subcommittee. Inv Ophthal Vis Sci 2013;54: TFOS37-70.

3. Young G. Evaluation of Soft Contact Lens Fitting Characteristics. Optom Vis Sci 1996;73:247254.

4. Bibby MM. Sagittal depth considerations in the selection of the base curve radius of a soft contact lens. Am J Optom Vis Sci 1979;56:407-13.

5. Garner LF. Sagittal height of the anterior eye and contact lens fitting. Am J Optom Physiol Opt 1982;59:301-5.

6. Snyder AC. Cosmetic extended-wear lenses: considering sagittal differences between the lens and eye. Int Cont Lens Clinic 1984;11:613-24.

7. Young G. Ocular sagittal height and soft contact lens fit. J Brit Cont Lens Assoc 1992;15:45-9.

8. van der Worp E, Mertz C. Sagittal height differences of frequent replacement silicone hydrogel contact lenses. Cont Lens Anterior Eye. 2015;38:157-62.

9. Kikkawa Y. Kinetics of soft contact lens fitting. Contacto 1979;23(Jul):10-17.

10. Hall L, Hunt C, Young G, Wolffsohn J. Factors affecting corneoscleral topography. Invest Ophthal Vis Opt 2013;54:3691-701.

11. Martin DK, Holden BA. A new method for measuring the diameter of the in vivo human cornea. Am J Optom Physiol Opt 1982;59:436-41.

12. Plainis S, Charman WN. On-eye power characteristics of soft contact lenses. Optom Vis Sci 1998;75:44-54.

13. Benjamini $Y$, Hochberg $Y$. Controlling the false discovery rate: a practical and powerful approach to multiple testing. J Royal Stat Soc Series B (Methodological) 1995;57:289-300.

14. Young G, Potts M, Sulley A. The effect of temperature on soft contact lens diameter. Eye Cont Lens 2016;42:298-302.

15. Ozkan J, Ehrmann K, Meadows D, Lally J, Holden B, de la Jara PL. Lens parameter changes under in vitro and ex vivo conditions and their effect on the conjunctiva. Cont Lens Anterior Eye 2013;36:171-5.

16. Young G, Coleman S. Poorly fitting soft lenses adversely affect ocular integrity. Cont Lens Assoc Ophthalmol J 2001;27:67-74.

17. Meier D. Das corneo-skleral-profil - ein kriterium individueller kontactlinsenanpassung. Die Kontaklinse 1992;26(10):4-11.

18. Hall L, Young G, Wolffsohn J, Riley C. The influence of corneo-scleral topography on soft contact lens Fit. Invest Ophthal Vis Opt 2011;52: 6801-6 
19. Young G, Schnider C, Hunt C, Efron S. Corneal topography and soft contact lens fit. Optom Vis Sci 2010;87:358-66.

20. Martin DK, Boulos J, Gan J, Gavriel K, Harvey P. A unifying parameter to describe the clinical mechanics of hydrogel contact lenses. Optom Vis Sci 1989;66:87-91.

21. Jenkins JT, Shimbo M. The distribution of pressure behind a soft contact lens. J Biomech Eng 1984;106:62-5.

22. Chauhan A, Radke CJ. Settling and deformation of a thin elastic shell on a thin fluid layer lying on a solid surface. J Colloid Interface Sci. 2002;245:187-97.

23. Hickson-Curran S, Young G, Brennan N, Hunt C. Chinese and Caucasian ocular topography and soft contact lens fit. Clin Exp Optom 2016;99:149-156.

24. Wang J, Jiao S, Ruggeri Cont Lens M, Shousha MA, Chen Q. In situ visualization of tears on contact lens using ultra high resolution optical coherence tomography. Eye Contact Lens. 2009;35:44-9.

25. Turhan SA, Toker E. Optical coherence tomography to evaluate the interaction of different edge designs of four different silicone hydrogel lenses with the ocular surface. Clin Ophthalmol. 2015;9:935-42. 


\section{Legends for Figures:}

Fig. 1: Schematic diagram of lens model showing lens strain and corneal overlap when fitted to the ocular surface

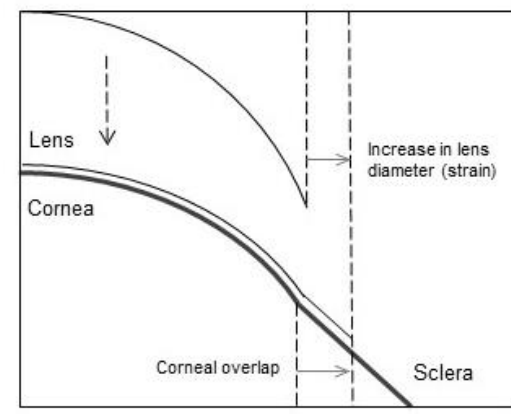

Fig. 2: Frequency distribution of edge strain (tightness) for an optimal design (8.60/14.2 mm BC/Diameter)

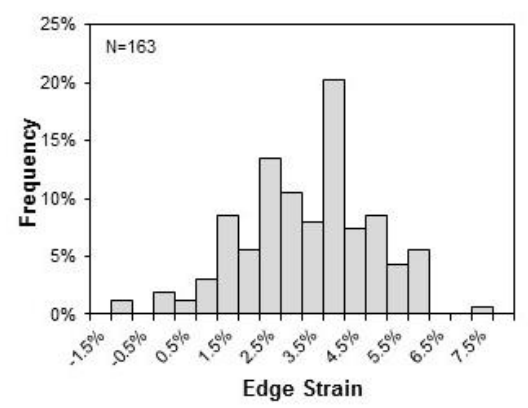

Fig. 3: Frequency distribution of corneal overlap (diameter acceptance) for an optimal design (8.60/14.2 mm BC/Diameter)

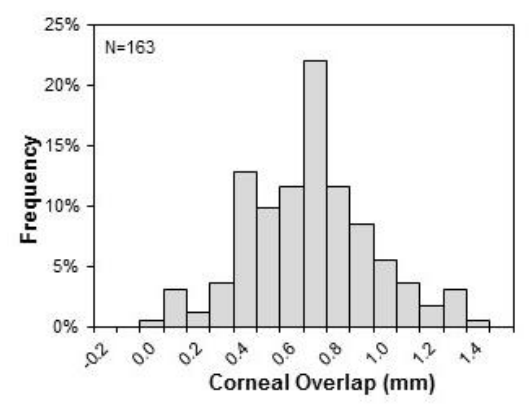

Fig. 4: Scatterplot of edge strain (tightness)) versus diameter acceptance (corneal overlap) for an optimal design (8.60/14.2 mm BC/Diameter) 


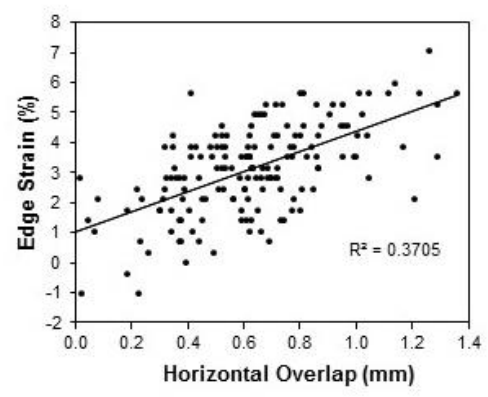

Fig. 5: Scatterplot of edge strain (tightness)) versus horizontal corneal asphericity (shape factor, $\left.1-\mathrm{e}^{2}\right)$ for an optimal design (8.60/14.2 mm BC/Diameter)

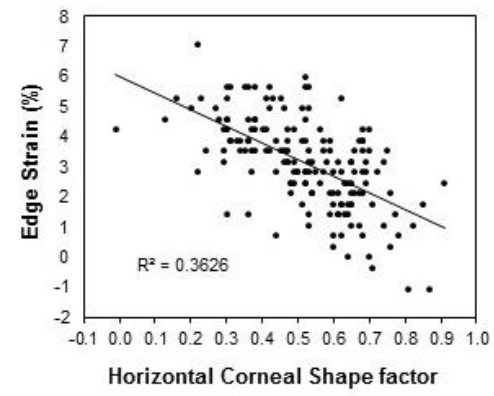

Fig. 6: Scatterplot of edge strain versus corresponding change in lens sagittal depth (8.60/14.2 $\mathrm{mm} \mathrm{BC/Diameter,} \mathrm{n}=164$ )

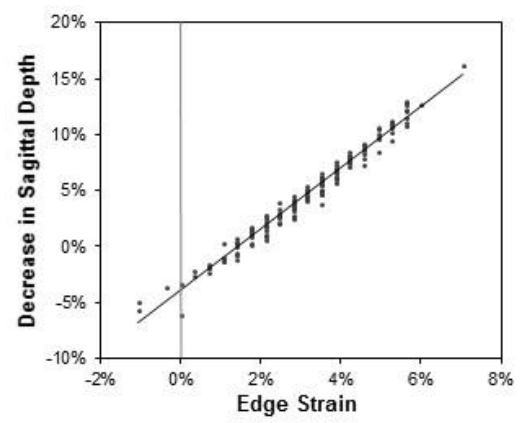

Fig. 7: Lenses of equivalent on-eye diameter for a typical eye. Shaded area indicates typical lens designs.

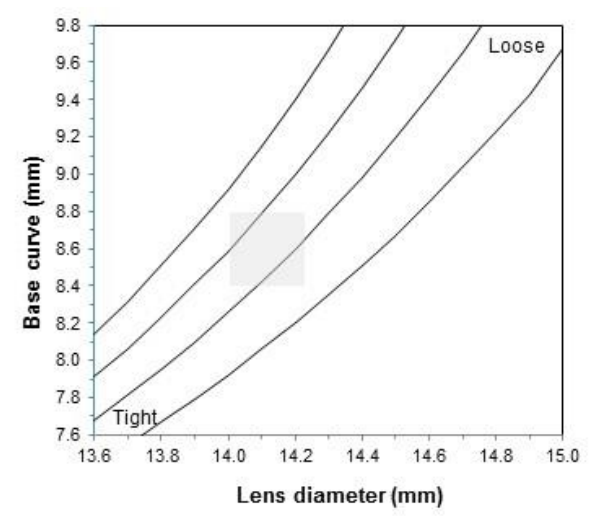


Fig. 8: $\quad$ Lenses of equivalent tightness for a typical eye. Percentage values indicate edge strain $(3 \%=$ optimum $)$. Shaded area indicates typical lens designs.

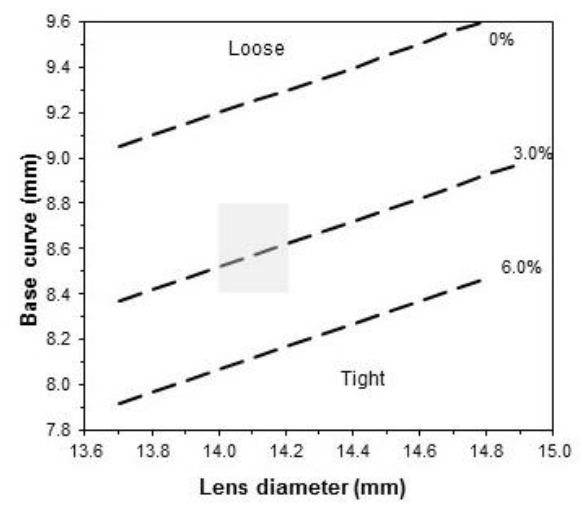

Fig. 9: Effect of lens asphericity $\left(1-\mathrm{e}^{2}\right)$ and sagittal depth on arclength. Lines indicate lenses of equivalent sagittal depth (Sag: $3.8 \mathrm{~mm} \equiv 8.35 / 14.0 \mathrm{~mm}$ ).

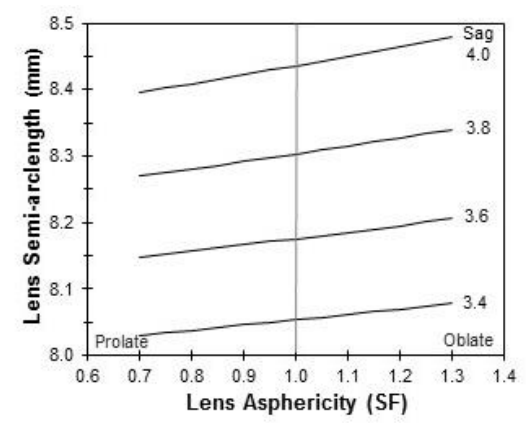


Table 1: Summary of ocular topography data $(\mathrm{N}=163)$

\begin{tabular}{|c|c|c|c|c|c|}
\hline \multirow{2}{*}{\multicolumn{2}{|c|}{ Ocular variable }} & \multicolumn{2}{|c|}{ Horizontal } & \multicolumn{2}{|c|}{ Vertical } \\
\hline & & Mean (SD) & Range & Mean (SD) & Range \\
\hline \multirow[t]{2}{*}{ Apical radius } & $(\mathrm{mm})$ & $7.78(0.30)$ & 7.01-8.77 & $7.74(0.30)$ & $6.94-8.67$ \\
\hline & (D) & 43.38 & 48.13-38.5 & & \\
\hline \multicolumn{2}{|l|}{ Shape factor } & $0.52(0.16)$ & $0.01-0.91$ & $0.80(0.15)$ & $0.17-1.44$ \\
\hline \multicolumn{2}{|c|}{ Corneal diameter (mm) } & $13.39(0.44)$ & $12.10-14.41$ & $13.10(0.67)$ & $11.18-14.41$ \\
\hline \multicolumn{2}{|c|}{ Corneoscleral junction angle $\left({ }^{\circ}\right)$} & $175.4(2.3)$ & 166.2-179.7 & $177.7(1.4)$ & $173.1-181.0$ \\
\hline
\end{tabular}

Table 2: Correlations between lens fit and ocular topography ( $\mathrm{N}=163)$. P-values incorporate a Benjamini and Hochberg correction for multiple comparisons.

\begin{tabular}{|l|c|c|c|c|}
\hline & \multicolumn{2}{|c|}{ Edge strain - average } & \multicolumn{2}{c|}{ Corneal overlap - horizontal } \\
\hline & $\mathbf{r}$ & $\mathbf{p}$-value & $\mathbf{r}$ & $\mathbf{p}$-value \\
\hline Corneal apical radius - mean & 0.521 & $<\mathbf{0 . 0 0 0 1}$ & -0.098 & 0.24 \\
\hline $\begin{array}{l}\text { Corneal asphericity (shape } \\
\text { factor) }\end{array}$ & -0.602 & $<\mathbf{0 . 0 0 0 1}$ & -0.419 & $<\mathbf{0 . 0 0 0 1}$ \\
\hline Corneal diameter - horizontal & -0.302 & $\mathbf{0 . 0 0 0 1}$ & -0.923 & $<\mathbf{0 . 0 0 0 1}$ \\
\hline Corneoscleral junction angle & -0.125 & 0.11 & -0.035 & 0.65 \\
\hline Ocular sag & -0.901 & $<\mathbf{0 . 0 0 0 1}$ & -0.546 & $<\mathbf{0 . 0 0 0 1}$ \\
\hline
\end{tabular}


Table 3: Overall success rates by lens diameter and base curve (assumes no shrinkage)

\begin{tabular}{|ccccccccc|}
\hline $\begin{array}{c}\text { Base curve } \\
(\mathbf{m m})\end{array}$ & $\mathbf{1 3 . 4}$ & $\mathbf{1 3 . 6}$ & $\mathbf{1 3 . 8}$ & $\mathbf{1 4 . 0}$ & $\mathbf{1 4 . 2}$ & $\mathbf{1 4 . 4}$ & $\mathbf{1 4 . 6}$ & $\mathbf{1 4 . 8}$ \\
\hline $\mathbf{8 . 0}$ & $51.5 \%$ & $57.7 \%$ & $49.7 \%$ & $28.8 \%$ & $20.2 \%$ & $8.6 \%$ & $2.5 \%$ & $0.6 \%$ \\
\hline $\mathbf{8 . 2}$ & $51.5 \%$ & $66.9 \%$ & $73.6 \%$ & $64.4 \%$ & $55.8 \%$ & $35.0 \%$ & $18.4 \%$ & $6.7 \%$ \\
\hline $\mathbf{8 . 4}$ & $36.8 \%$ & $60.7 \%$ & $77.9 \%$ & $83.4 \%$ & $81.0 \%$ & $69.9 \%$ & $50.9 \%$ & $26.4 \%$ \\
\hline $\mathbf{8 . 6}$ & $28.2 \%$ & $50.9 \%$ & $69.3 \%$ & $84.7 \%$ & $90.2 \%$ & $87.7 \%$ & $77.9 \%$ & $58.3 \%$ \\
\hline $\mathbf{8 . 8}$ & $20.9 \%$ & $36.2 \%$ & $55.8 \%$ & $73.6 \%$ & $86.5 \%$ & $87.7 \%$ & $85.9 \%$ & $73.6 \%$ \\
\hline $\mathbf{9 . 0}$ & $16.6 \%$ & $25.2 \%$ & $43.6 \%$ & $61.3 \%$ & $74.2 \%$ & $82.2 \%$ & $84.0 \%$ & $80.4 \%$ \\
\hline $\mathbf{9 . 2}$ & $9.8 \%$ & $16.6 \%$ & $25.2 \%$ & $46.0 \%$ & $59.5 \%$ & $69.3 \%$ & $75.5 \%$ & $74.8 \%$ \\
\hline $\mathbf{9 . 4}$ & $7.4 \%$ & $11.0 \%$ & $16.0 \%$ & $27.6 \%$ & $41.7 \%$ & $54.0 \%$ & $62.0 \%$ & $65.0 \%$ \\
\hline $\mathbf{9 . 6}$ & $4.3 \%$ & $6.1 \%$ & $9.8 \%$ & $18.4 \%$ & $25.2 \%$ & $31.9 \%$ & $43.6 \%$ & $45.4 \%$ \\
\hline
\end{tabular}

